\title{
ARTICLE
}

\section{Design and operation of the H4IRRAD mixed-field test area at CERN}

\author{
Bartolomej Biskup $^{\mathrm{a}, \mathrm{b}^{*}}$, Markus Brugger ${ }^{\mathrm{a}}$, Marco Calviani ${ }^{\mathrm{a}}$, Ilias Efthymiopoulos ${ }^{\mathrm{a}}$ and Regina Kwee ${ }^{\mathrm{a}}$ \\ ${ }^{a}$ CERN, Geneva 23, 1211, Switzerland; ${ }^{b}$ Czech Technical University in Prague, Prague, 16636, Czech republic
}

\begin{abstract}
H4IRRAD is a new mixed-field irradiation area at CERN, designed for testing LHC electronic equipment, especially large volume assemblies such as full electronics racks of high current power converters. The area uses alternatively an attenuated primary $400 \mathrm{GeV} / \mathrm{c}$ proton beam slowly extracted from the CERN SPS, or a secondary, mainly proton, beam of $280 \mathrm{GeV} / \mathrm{c}$ directed towards a $1 \mathrm{~m}$ long, $7.5 \mathrm{~cm}$ diameter $\mathrm{Cu}$ target. Different shielding layers are used to reproduce a radiation field similar to the LHC "tunnel" and "shielded" areas in test zones around the target. The FLUKA Monte Carlo code was used for the design and optimization studies with the objective to match, as closely as possible, the mixed-radiation fields in the LHC ring, whilst also maintaining the lowest possible field gradient within the irradiation areas. Activation studies for the target, equipment, and the air volume were performed in order to assess safety risks, specify the access conditions and determine how to handle the equipment after irradiation. The test area has been in operation since 2011 with numerous tests performed already. The design concept of H4IRRAD, along with comparison studies between the FLUKA predictions and the measurements for the mixed radiation field characterization will be presented in this paper.
\end{abstract}

Keywords: FLUKA; high energy hadron fluence; thermal neutron fluence; dose; Si 1 MeV neutron equivalent fluence; mixed-field calibration; RadMons; BLM; LHC equipment testing

\section{Introduction}

LHC operation has proven to be highly successful, with relatively high uptime for physics. This achievement has been accompanied by system and control failures of the electronic equipment along the LHC tunnel due to radiation, mainly in the form of Single Event Effects (SEE). However, in the future with increased machine luminosity, the subsequent risk from the failures of equipment due to the integrated radiation has to be minimized. The lack of experimental data concerning these radiation-induced failures on specific and mostly commercial equipment required the creation of a dedicated facility where such studies could be performed in a well-controlled environment and in a sufficiently short time schedule.

H4IRRAD [1] is a new irradiation area at CERN for testing LHC electronic equipment, especially large volume assemblies such as full-size electronics racks of high current power converters. The test area is located in the H4 beam line in the North Experimental Area of CERN. It uses alternatively an attenuated primary $400 \mathrm{GeV} / \mathrm{c}$ proton beam slowly extracted (flat top of $10 \mathrm{~s}$ ) from the CERN SPS, or a secondary, mainly proton, beam of $280 \mathrm{GeV} / \mathrm{c}$, directed towards an H4IRRAD $\mathrm{Cu}$ ( $1 \mathrm{~m}$ long, $7.5 \mathrm{~cm}$ diam.) target. The test area provides several locations around the $\mathrm{Cu}$ target,

*Corresponding author. Email: bartolomej.biskup@cern.ch separated by different shielding layers, which allow the reproduction of a radiation field similar to the LHC "tunnel" and "shielded" areas. The high energy hadron (HEH) fluence, particle type and energy spectra shape in the test locations are matched as closely as possible to those found in the LHC, which allows the reproduction of the same failure types (particle and energy dependent) as those observed in the LHC machine. Dose maps and the $1 \mathrm{MeV}$ Si neutron equivalent fluence maps are also matched.

For design and optimization studies, as well as radiation field predictions, the FLUKA Monte Carlo code [2-3] was employed.

\section{Layout and generic studies}

The H4IRRAD layout is shown in Figure 1 with the two irradiation zones: the internal zone, located downstream the $\mathrm{Cu}$ target, and the external zone, separated by a concrete wall ("inner shielding"). The internal irradiation area corresponds to the LHC "tunnel" locations, while the external irradiation area to the LHC "shielded" areas.

For the first studies, a simplified geometry of H4IRRAD was simulated by FLUKA, assuming a beam of $320 \mathrm{GeV} / \mathrm{c}$ and $10^{9}$ protons over $44 \mathrm{~s}$ (giving approximately $1.375 \cdot 10^{13} \mathrm{p} /$ week), corresponding to an 
SPS supercycle. To optimize the radiation field in the external test location, the influence of the inner shielding material composition and thickness was studied. By maximizing the HEH fluence and matching the neutron spectra shapes to those of the LHC, the most promising setups were found, and are listed in Table 1, together with examples of the LHC tunnel and shielded areas.

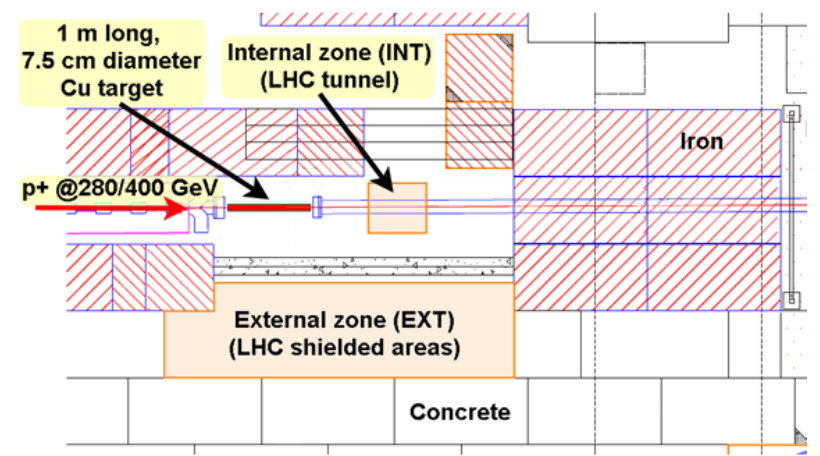

Figure 1. Layout of the H4IRRAD irradiation areas. The hatched areas indicate the iron blocks of the shielding while the white ones are concrete blocks. The beam enters from the left of the picture. The overall shielding around the beam line is not shown in this excerpt.

Table 1. HEH and neutron fluence fractions for various inner shielding setups (iron + concrete, concrete, polyethylene and no shielding), and examples of LHC areas. $\Phi_{\text {nth }}-$ thermal neutron fluence, $\Phi_{\mathrm{HEn}}-$ high energy $(>20 \mathrm{MeV})$ neutron fluence, $\Phi_{5-20 \mathrm{MeV}}-5-20 \mathrm{MeV}$ neutron fluence, $\Phi_{\mathrm{HEH}}-$ all high energy hadrons fluence. The test positions in the simplified geometry don't correspond to the final ones, therefore the results are not comparable with the data in Table 2.

\begin{tabular}{ccccc}
\hline Setup & Location & $\begin{array}{c}\Phi_{\text {nth }} \\
/ \Phi_{\mathrm{HEn}}\end{array}$ & $\begin{array}{c}\Phi_{5-20 \mathrm{MeV}} \\
/ \Phi_{\mathrm{HEn}}\end{array}$ & $\begin{array}{c}\Phi_{\mathrm{HEH}} \\
/ \text { week } / \mathrm{cm}^{2}\end{array}$ \\
\hline $20 \mathrm{~cm} \mathrm{Fe}+$ & INT & 1.1 & 0.53 & $5.1 \mathrm{E}+10$ \\
\cline { 2 - 5 } $20 \mathrm{~cm} \mathrm{Con}$ & EXT & 12.1 & 0.34 & $7.3 \mathrm{E}+08$ \\
\hline $20 \mathrm{~cm} \mathrm{Con}$ & EXT & 5.0 & 0.45 & $2.8 \mathrm{E}+09$ \\
\hline $10 \mathrm{~cm} \mathrm{PE}$ & EXT & 3.3 & 0.44 & $3.9 \mathrm{E}+09$ \\
\hline No Sh & EXT & 5.3 & 0.81 & $4.7 \mathrm{E}+09$ \\
\hline LHC & IR1 Q6 - tun. & 5.6 & 0.48 & \\
\cline { 2 - 5 } & IR1- RR13/17 & 26.5 & 0.46 & \\
\hline
\end{tabular}

The setup combining an iron and concrete shielding seems to have the spectrum most similar to the spectra in LHC shielded areas such as IR1-RR13/17. However, the $\mathrm{HEH}$ fluence is a factor of 5 lower than for the pure polyethylene (PE) shielding setup. Finally the $20 \mathrm{~cm}$ concrete inner shielding was deemed a suitable compromise between achieving a maximum HEH fluence, particle spectra shape and the HEH gradient in the test locations, and was therefore chosen for the final configuration.

\section{Particle spectra and radiation levels}

In Figure 2 and Figure 3 the simulated energy spectra of neutrons at the H4IRRAD internal (external) locations are compared with those of the LHC tunnel (shielded) areas. For H4IRRAD a "nominal" beam of $280 \mathrm{GeV} / \mathrm{c}$ and $10^{9}$ protons per $44 \mathrm{~s}$ SPS cycle is used. The ratio between thermal and high energy neutrons is well reproduced (Figure 2). Figure 3 shows that at H4IRRAD external positions the thermal neutron component is lower than in the shielded LHC locations, due to the combined concrete/iron shielding employed, but still well adapted for the scope of the tests.

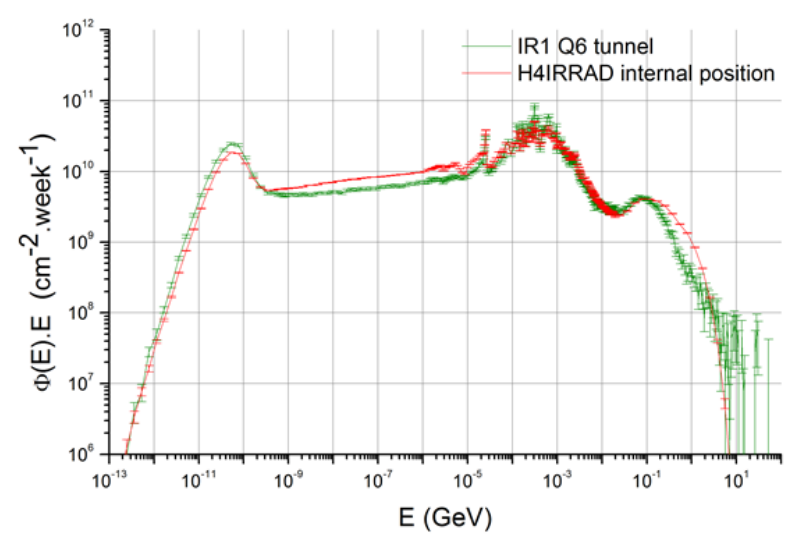

Figure 2. H4IRRAD neutron spectra (internal location) in comparison with those of the LHC "tunnel" IR1-Q1 location. Both spectra are normalized at the $100 \mathrm{MeV}$ point.

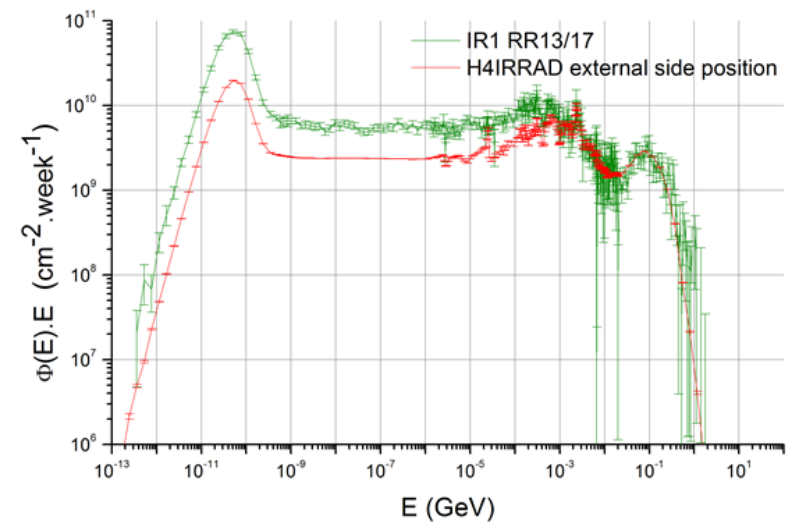

Figure 3. H4IRRAD neutron spectra (external location) in comparison with those of the LHC "shielded" IR1-RR13/17 location. Both spectra are normalized at the $100 \mathrm{MeV}$ point.

Table 2. Expected prompt dose (in air), HEH fluence $\left(\Phi_{\mathrm{HEH}}\right)$, and $\mathrm{Si} 1 \mathrm{MeV}$ neutron equivalent fluence $\left(\Phi_{\text {neq }}\right)$. The values are normalized per week of operation with the nominal beam of $280 \mathrm{GeV}$.

\begin{tabular}{cccc}
\hline Test location & $\begin{array}{c}\text { Absorbed } \\
\text { dose }(\mathrm{Gy})\end{array}$ & $\begin{array}{c}\Phi_{\text {HEH }} \\
\left(10^{9} / \mathrm{cm}^{2}\right)\end{array}$ & $\begin{array}{c}\Phi_{\text {neq }} \\
\left(10^{9} / \mathrm{cm}^{2}\right)\end{array}$ \\
\hline Internal & 13.6 & 22.1 & 102 \\
External side & 2.48 & 6.76 & 30.5 \\
Ext.- downstream & 2.57 & 6.22 & 19.9 \\
\hline
\end{tabular}

Typical absorbed dose and particle fluences were simulated using FLUKA and are shown in Table 2. The radiation field gradient in the internal and external test zones for the high energy hadron fluence profile for ordinary rack-size equipment is expected to be lower than $30 \%$. The strongest influence is expected to be on 
the vertical (horizontal) axis at the internal (external) test locations.

\section{Radiation protection studies}

With regards to radiation protection and safety, and, eventually, for defining access conditions during operation, the most critical task was to evaluate the prompt dose outside the area; the air activation of the area and residual dose rate of equipment are also critical for specifying the access conditions into the target zone and for handling the equipment after the irradiation period.

Several iterations were performed to define the final layout of the area and satisfy all safety requirements: the outside concrete shielding had to be prolonged in critical accessible areas to assure a dose equivalent rate below the allowed limits (see below), and particular care was taken to displace or shield cable passages and other openings in the shielding in order to limit the streaming of thermal neutrons to the outside zones.

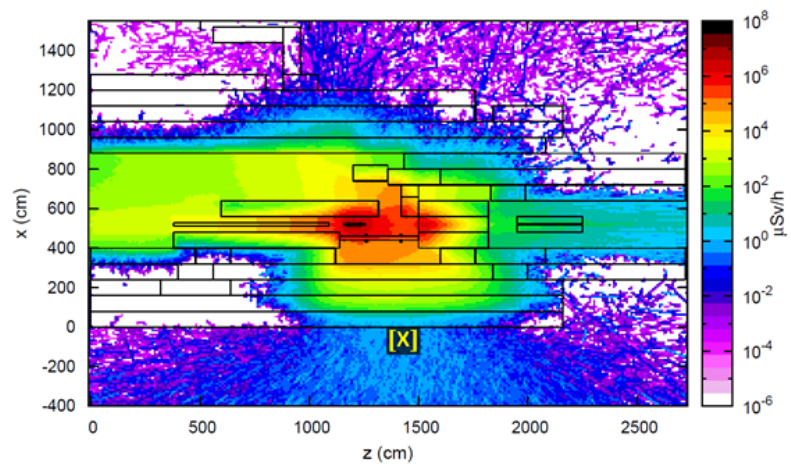

Figure 4. Horizontal projection of simulated average prompt dose equivalent in $\mu \mathrm{Sv} / \mathrm{h}$ averaged over $30 \mathrm{~cm}$ around a beam height for the H4IRRAD layout. At the position X, the expected dose equivalent was evaluated (see text below).

Figure 4 shows the average prompt dose equivalent $\left(H^{*}(10)\right)$ inside and around H4IRRAD with a beam of $10^{9} 280 \mathrm{GeV} / \mathrm{c}$ protons every $44 \mathrm{~s}$. The expected dose equivalent at the position $\mathrm{X}$, which is located in accessible, non-permanent work area, is $0.6 \pm 6 \% \mu \mathrm{Sv} / \mathrm{h}$, well below the allowed limit of a low-occupancy supervised radiation area of $15 \mu \mathrm{Sv} / \mathrm{h}$ [4], which is the classification of the North Area Experimental Hall. The maximal prompt dose equivalent above the roof can reach up to $100 \mu \mathrm{Sv} / \mathrm{h}$ at the opening of the cable passage. Therefore, access to the top location is blocked during operation of H4IRRAD [5].

The average muon fluence directly behind the H4IRRAD iron beam dump is $3.3 \cdot 10^{6}$ muons $/ \mathrm{cm}^{2} /$ week, corresponding to a dose equivalent of $6.2 \mu \mathrm{Sv} / \mathrm{h}$. This location is still inside the beamline area, thus not accessible during operation.

The air activation around the H4IRRAD target zone was studied taking a conservative estimate of the air volume around the $\mathrm{Cu}$ target, using both test areas and the tunnel volume up to $11 \mathrm{~m}$ upstream. After two weeks of continuous operation with the nominal beam of $280 \mathrm{GeV} / \mathrm{c}$, and assuming the worst case scenario, i.e. no air leakage or exchanging during the irradiation and no air flushing after the irradiation, the average air activation rate would decrease to under $1 \mu \mathrm{Sv} / \mathrm{h}$ after approximately 5 minutes of cooling time.

Table 3 summarizes the residual dose equivalent of the H4IRRAD instrumentation and test equipment for various cooling times. The $\mathrm{Cu}$ target and its support table have non-negligible activation. Therefore special pre-defined handling options have been studied and $\mathrm{aPb}$ shielding is used for 2012 operations $(400 \mathrm{GeV} / \mathrm{c}$ and $3 \cdot 10^{9}$ protons per $44 \mathrm{~s}$ SPS cycle) prior to interventions close to the target to limit its contribution to the ambient dose during access to the area.

Table 3. Residual dose equivalent (skin to material contact) for H4IRRAD instrumentation and test equipment after several cooling times, assuming 20 days of irradiation with nominal beam conditions.

\begin{tabular}{cccc}
\hline & \multicolumn{3}{c}{ Residual dose equivalent $(\mu \mathrm{Sv} / \mathrm{h})$} \\
Cooling time & 1hour & 1day & 1month \\
\hline Target & 218 & 104 & 31.1 \\
Table & 160 & 52.2 & 0.73 \\
Dump & 8.66 & 4.38 & 0.79 \\
Int circ. & 6.74 & 3.08 & 0.73 \\
Ext circ. & 3.22 & 1.17 & 0.22 \\
\hline
\end{tabular}

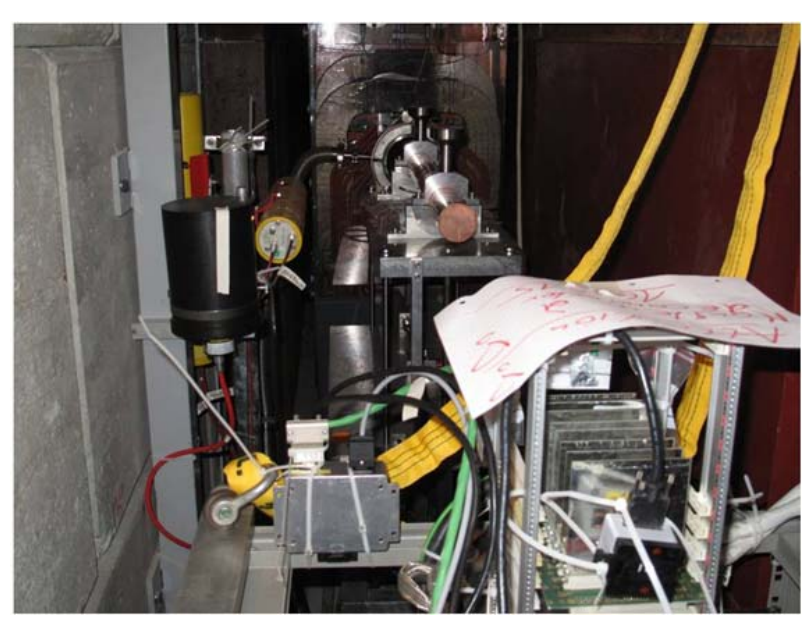

Figure 5. Photo of the internal location with the $\mathrm{Cu}$ target, the monitoring detectors and the test equipment.

\section{Operation of the H4IRRAD area}

The H4IRRAD was built in a short period to cover the needs of LHC in testing large volume electronic assemblies using a beam of reduced intensity to ease the accessibility for the users, compared to other high-intensity facilities like CNRAD [6], for which the access is also linked to the CNGS beam to Gran Sasso. Two special platforms, able to carry the heavy test equipment, were designed in order to permit their lowering into the internal (see Figure 5) and external test areas from the top, using the overhead crane of the building. This semi-remote operation allows for the 
exchange of user equipment with limited access to the irradiation areas and resulting exposure to radiation. The H4IRRAD test area started operation with first tests in June 2011, using a secondary beam of $280 \mathrm{GeV} / \mathrm{c}$ protons.

During commissioning a set of scintillators and two types of ionization chambers were used to create a coincidence trigger to cross-calibrate and provide the number of protons hitting the H4IRRAD target $\left(\mathrm{N}_{\text {pot }}\right)$. The $\mathrm{N}_{\text {pot }}$ and the beam profile $(24 \times 2.3 \mathrm{~mm}$ FWHM disturbed Gaussian for 2011 and $7 \times 4 \mathrm{~mm}$ FWHM Gaussian for 2012 operation), also measured using filament scanner and wire chambers, are used as a normalization input for the FLUKA simulations.

The mixed-field components are measured using dedicated radiation monitors for electronic systems (RadMons) and beam-loss monitors (BLMs), of the same types as used in the LHC.

RadMons [7], based on SRAM memory device, are used as Single Event Upset (SEU) monitors. Their response to SEU is estimated using: $N_{S E U}=\sigma_{n t h} \cdot \Phi_{n t h}+$ $\sigma_{H E H} \cdot \Phi_{H E H}$, where $\sigma$ and $\Phi$ denote the failure cross-section and fluence of thermal neutrons and $\mathrm{HEH}$, respectively. The cross-sections depend on a voltage bias of the RadMon and can be found in Ref. [8].

Table 4 shows an example of comparison between fluences calculated from measured $\mathrm{N}_{\mathrm{SEU}}$ (by several RadMons operated during one irradiation slot on two different biases) and respective fluences simulated with FLUKA at the RadMon positions.

Table 4. Comparison between measured and simulated HEH and thermal neutron fluences per proton on target (p.o.t.) for different RadMon positions (RM1, RM5 in the external location, RM4 in the internal one). The high experimental data errors are dominated by the possible variation of cross-section between memory chips. The statistical errors of simulations are negligible, but the position uncertainty could extend these errors up to $\sim 10 \%$.

\begin{tabular}{|c|c|c|c|}
\hline Detector & \multicolumn{3}{|c|}{$\Phi_{\mathrm{HEH}}\left(10^{-3} /\right.$ p.o.t. $\left./ \mathrm{cm}^{2}\right)$} \\
\hline RM1 & $0.37 \pm 43 \%$ & $0.45 \pm 1.1 \%$ & $1.20 \pm 43 \%$ \\
\hline RM4 & $1.89 \pm 43 \%$ & $1.96 \pm 0.6 \%$ & $1.04 \pm 43 \%$ \\
\hline RM5 & $0.69 \pm 43 \%$ & $0.85 \pm 0.8 \%$ & $1.23 \pm 43 \%$ \\
\hline & \multicolumn{3}{|c|}{$\Phi_{\text {nth }}\left(10^{-3} /\right.$ p.o.t. $\left./ \mathrm{cm}^{2}\right)$} \\
\hline RM1 & $0.99 \pm 43 \%$ & $2.90 \pm 0.5 \%$ & $2.9 \pm 43 \%$ \\
\hline RM4 & $1.21 \pm 43 \%$ & $3.29 \pm 0.4 \%$ & $2.7 \pm 43 \%$ \\
\hline RM5 & $0.99 \pm 43 \%$ & $2.87 \pm 0.4 \%$ & $2.9 \pm 43 \%$ \\
\hline
\end{tabular}

The simulated values of $\Phi_{\mathrm{HEH}}$ are slightly overestimated, while values of $\Phi_{\text {nth }}$ are overestimated by a factor of 2 to 3 when comparing to the data, due to the unknowns related to the concrete and iron composition in the shielding, as well as the altering of the neutron spectra due to the presence of test equipment.

The type of BLM used in the LHC is a cylindrical parallel-plate ionization chamber. In H4IRRAD two such BLMs are installed at beam height: one horizontally aligned, the other vertically aligned. Their location close to the target (with a distance of 34 and
$55 \mathrm{~cm}$, respectively) coupled with their size makes them very sensitive to the gradient of the radiation field. The measured dose corrected for the time-dependent offset has been integrated over different time periods and compared with the dose simulated at the BLM positions. For the horizontal BLM, the simulated and measured data are in agreement within $15 \%$, while for the vertical one the differences are up to $30 \%$, probably due to the fact that the vertical BLM is partially shielded by the horizontal BLM and their support, which is not considered in the simulation.

In 2012, H4IRRAD beam operated using an attenuated primary proton beam of $400 \mathrm{GeV} / \mathrm{c}$ and a factor three higher intensity. The different energy and higher intensity have minimal impact on the radiation field spectra, while contributing significantly to the efficient use of the irradiation area.

\section{Conclusion}

A new test area was designed and put in operation in June 2011 in the H4 beam line of CERN SPS North Area Experimental Hall. The new area offers test locations with mixed field radiation spectra similar to those of the LHC tunnel areas. The radiation field was simulated with FLUKA and compared to measurements obtained from RadMons as well as BLMs, yielding a good agreement, well suited for the scope of the tests.

From the first test campaigns, H4IRRAD has been identified as a key test area that can provide vital feedback to electronic systems operated in the LHC as well as CERN's accelerator chain in general. Results on SEU and total ionizing dose on test equipment at H4IRRAD provide the necessary experimental data to understand the radiation-induced failures on specific equipment installed in the LHC machine, thus allowing the optimization of their operation and achieving maximum performance required to reach the LHC midand long-term physics goals.

\section{Acknowledgements}

The authors would like to thank all colleagues who contributed to the installation, commissioning and successful operation of the first H4IRRAD test campaigns, the CERN Radiation Protection team for their continuous and valuable help and support, as well as the R2E Project for the funding and organization of the radiation tests.

\section{References}

[1] https://espace.cern.ch/h4irrad/default.aspx.

[2] G. Battistoni, S. Muraro, P. R. Sala, F. Cerutti, A. Ferrari, S. Roesler, A. Fasso and J. Ranft, The FLUKA code: Description and benchmarking, Proceedings of the Hadronic Shower Simulation Workshop 2006, Fermilab 6--8 September 2006, M. Albrow, R. Raja eds., AIP Conference Proceeding 
896 (2007), pp. 31-49.

[3] A. Ferrari, P. R. Sala, A. Fasso and J. Ranft, FLUKA: A Multi-Particle Transport Code, CERN-2005-10 (2005), INFN/TC_05/11, SLAC-R-773.

[4] D. Forkel-Wirth and T. Otto, Area Classification, EDMS 810149, CERN (2006).

[5] B. Biskup, M. Brugger, M. Calviani, I. Efthymiopoulos, R. Kwee, J. Mekki, F. La Torre, E. Lebbos, P. Mala, G. Manessi, A. Nordt, F. Pozzi, K. Roeed, C. Severino, M. Silari and A. Thornton, Commissioning and Operation of the H4IRRAD Mixed-Field Test Area, CERN-ATS-Note-2011-121 PERF (2011).
[6] http://cern.ch/radwg

[7] G. Spiezia, J. Mekki, S. Batuca, M. Brugger, M. Calviani, A. Ferrari, D. Kramer, R. Losito, A. Masi, A. Nyul, P. Peronnard, C. Pignard, K. Roeed and T. Wijnands, The LHC accelerator Radiation Monitoring System - RadMON, 10th International Conference on Large Scale Applications and Radiation Hardness of Semiconductor Detectors, PoS(RD11)024 (2012).

[8] K. Roeed, V. Boccone, M. Brugger, A. Ferrari, D. Kramer, E. Lebbos, R. Losito, A. Mereghetti, G. Spiezia and R. Versaci, FLUKA simulations for SEE studies of critical LHC underground areas, IEEE Trans. Nucl. Sci. 58 (2011), pp. 932-938. 